\title{
Lightweight Mobile and Wireless Systems: Technologies, Architectures, and Services
}

\author{
Charalabos Skianis, ${ }^{1}$ Fabrizio Granelli, ${ }^{2}$ Periklis Chatzimisios, ${ }^{3}$ \\ Christos Verikoukis, ${ }^{4}$ and Michael Devetsikiotis ${ }^{5}$
}

${ }^{1}$ Department of Information and Communication Systems Engineering (ICSE), University of the Aegean, 81100 Mytilene, Greece
${ }^{2}$ Department of Information Engineering and Computer Science (DISI), University of Trento, 38123 Trento, Italy
${ }^{3}$ Department of Informatics, Alexander Technological Educational Institute of Thessaloniki, Thessaloniki, 574 00 Macedonia, Greece
${ }^{4}$ Centre Tecnologic de Telecomunicacions de Catalunya (CTTC), O8860 Barcelona, Spain
${ }^{5}$ North Carolina State University (NCSU), Raleigh, NC 27695, USA

Correspondence should be addressed to Charalabos Skianis, cskianis@aegean.gr

Received 31 December 2010; Accepted 31 December 2010

Copyright (C) 2010 Charalabos Skianis et al. This is an open access article distributed under the Creative Commons Attribution License, which permits unrestricted use, distribution, and reproduction in any medium, provided the original work is properly cited.

Wireless communications are becoming increasingly pervasive, as the number and diffusion of portable wireless equipped devices are exponentially increasing (ranging from cellular phones to handheld game consoles, from personal digital assistant and personal navigation devices to still and video cameras). This results in an unprecedented request for lightweight, wireless communication devices with high usability and performance able to support added-value services in a highly mobile environment. Such devices follow the users everywhere they go (at work, at home, while travelling, in a classroom, etc.) and result in exciting research, development, and business opportunities as evidenced from a plethora of currently undergoing European and national funded projects and consortiums (e.g., ICARUS, NEWCOM++, MIMAX, OMEGA, MOBILIA, REWIND, and SELFNET).

The above scenario clearly demands significant upgrades to the existing communication paradigm in terms of infrastructure, devices, and services to support the "anytime, anywhere, any device" philosophy, providing novel and fastevolving requirements and expectations on research and development in the field of information and communication technologies. The core issue is to support wireless users' desire for 24/7 network availability and transparent access to "their own" services.

This issue gathers together a selection of recent original research in the field comprising of six diverse papers that reflect the variety of concerns and latest advances relating to lightweight mobile and wireless systems.

In the first paper "Hidden Anchor: A Lightweight Approach for Physical Layer Location Privacy," R. El-Badry et al. propose an algorithm that provides anchor physical layer location privacy for different classes of localization algorithms in wireless sensor networks. The performance of the proposed algorithm is evaluated through analysis and simulation experiments and it is shown that it can hide the location and identity of anchor nodes with very low overhead and without limiting the localization accuracy for trusted nodes. The authors also provide a technique that significantly enhances the privacy of the network without affecting the localization accuracy at trusted nodes.

The second paper "Simulation of 802.21 Handovers Using $n s-2$ " by $\mathrm{H}$. Marques et al. presents a short description of the 802.21 standard, its implementation in ns-2 as well as the employed signaling in a handover between WiMAX and Wi-Fi networks. Moreover, the paper evaluates the reliability and scalability of ns-2 tool in simulating multiple vertical handover scenarios under the scope of IEEE 802.21 and also proposes a novel and very simple approach to determine the expected number of handovers in an ns-2 simulation.

In the paper "Service-Aware Retransmission Control in Cellular Networks," N. B. Halima et al. propose a serviceaware cross-layer approach between application/transport layers on the mobile terminal and link layer on the wireless 
base station to enable dynamic control on the level of per-packet hybrid ARQ (HARQ) protection for multimedia data streams. Experimental results demonstrate the potential benefits deriving from the proposed strategy, underlining relevant improvements for audio and video flows as well as for TCP-based data transfers.

In the paper "Crafting a Real-Time Information Aggregator for Mobile Messaging," J.-S. Leu develops a real-time information aggregator called visualizing SMS and MMS messages system (VSMMS) in order to visualize instant SMS/MMS messages openly on a larger device, instead of a limited-sized screen on personal cellular phones in the past. VSMMS includes an optional web-based interface to help the administrator verify the incoming contents and, thus, appears to be suitable for public mass media broadcasting and real-time information sharing.

The paper "Optimized Hybrid Resource Allocation in Wireless Cellular Networks with and without Channel Reassignment" by $\mathrm{X}$. Wu et al. presents two efficient integer linear programming formulations for the hybrid channel assignment (HCA) problem in wireless cellular networks. The proposed approaches optimally allocate a channel (from a pool of available channels) to an incoming call such that both hard (i.e., cosite and adjacent channel constraints) and soft constraints (i.e., the packing condition, resonance condition, and limiting channel reassignment) are satisfied.

Finally, in "Spatial Diversity Scheme to Efficiently Cancel ISI and ICI in OFDM-OQAM Systems," N. Zorba and F. Bader propose a spatial diversity scheme to cancel the intersymbol interference (ISI) and intercarrier interference (ICI) in the system through low-complexity operations in order to enable the implementation of an offset quadrature amplitude modulation (OQAM) orthogonal frequency division multiplexing (OFDM) transmission scheme. The authors formulate the performance of the proposed scheme in terms of data rate and BER and mathematically obtained the SNR expression. The performance results indicate that the OFDM-OQAM proposal increases the system data rate comparing to the classical cyclic prefix (CP) OFDM systems.

\section{Acknowledgments}

We would like to thank all the authors for contributing papers to this special issue and the external reviewers for their tireless work and efforts during the reviewing process. We also would like to thank the Editorial Staff of Journal of Computer Systems, Networks, and Communications for their continuous support in editing this special issue. This special issue has been organized with the support of the FP7 Network of Excelence NEWCOM++ (216715).

Charalabos Skianis Fabrizio Granelli

Periklis Chatzimisios

Christos Verikoukis

Michael Devetsikiotis 

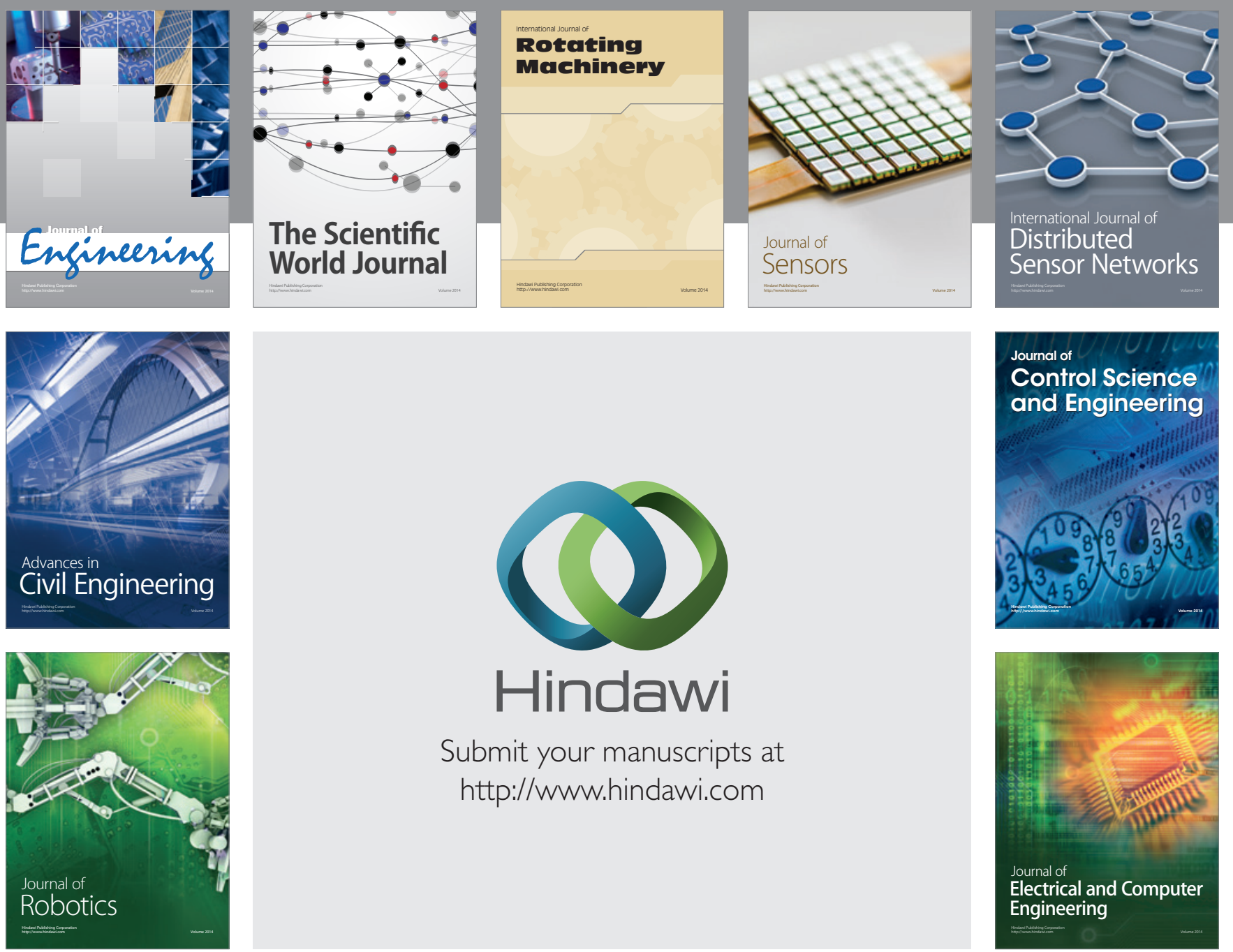

Submit your manuscripts at

http://www.hindawi.com
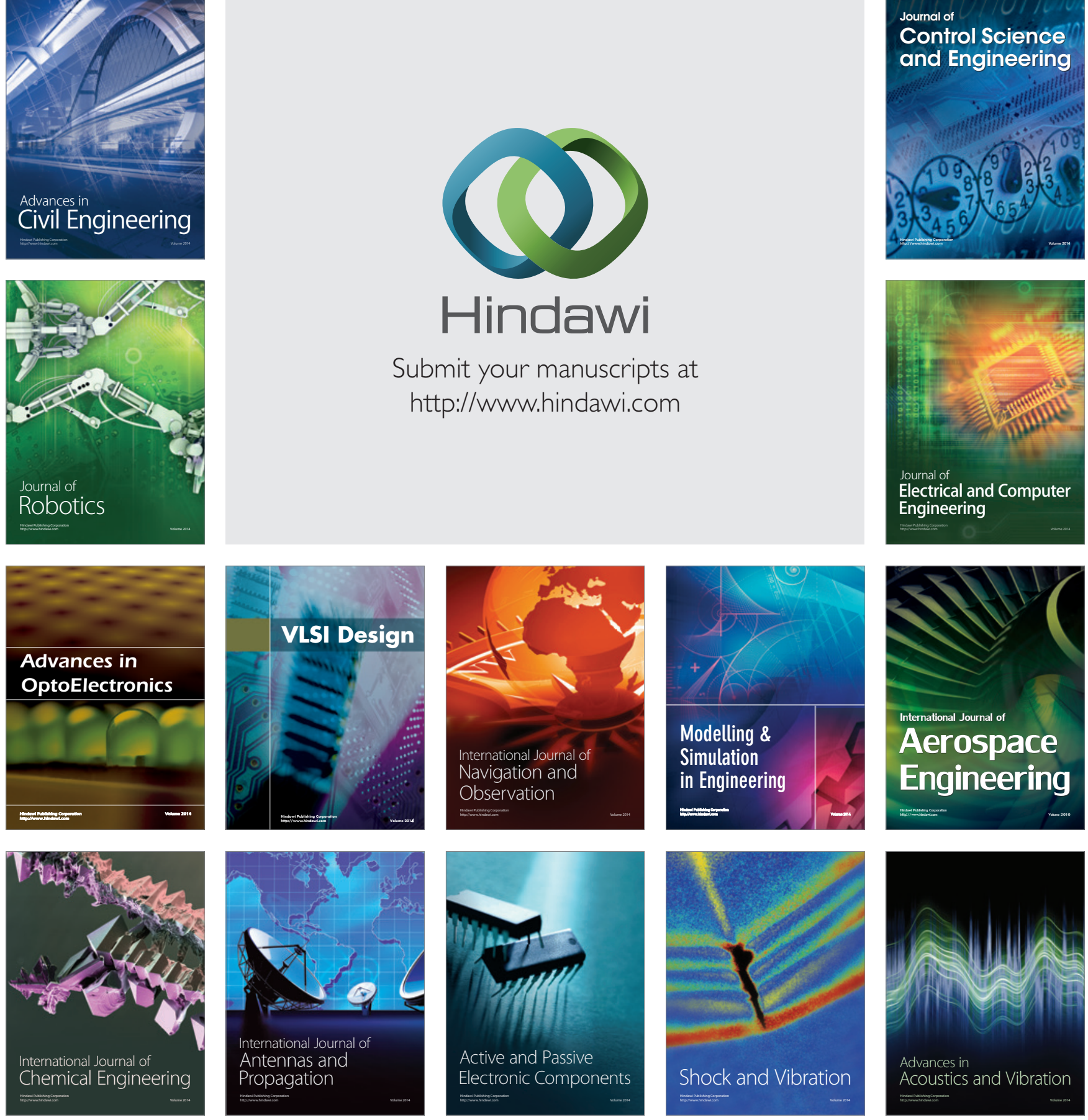